\title{
Management of mandibular odontogenic keratocyst through radical resection: Report of 35 cases
}

\author{
NYIMI BUSHABU FIDELE ${ }^{1,2}$, LIU BING ${ }^{1,2}$, YANFANG SUN ${ }^{1}$, TIANFU WU ${ }^{1}$, \\ YUEYU $_{\text {ZHENG }}{ }^{1}$ and YIFANG ZHAO $^{1,2}$ \\ ${ }^{1}$ Department of Oral Maxillofacial Head and Neck Oncology Surgery, ${ }^{2}$ The State Key Laboratory Breeding Base \\ of Basic Science of Stomatology, and Key Laboratory of Oral Biomedicine Ministry of Education, \\ School and Hospital of Stomatology, Wuhan University, Wuhan, Hubei 430079, P.R. China
}

Received August 18, 2018; Accepted April 15, 2019

DOI: $10.3892 / \mathrm{ol} .2019 .10367$

\begin{abstract}
The present study reported the clinical outcomes of 35 patients with mandibular odontogenic keratocysts (OKCs) following treatment by radical resection and immediate defect reconstruction. Amongst 565 patients with OKCs that were treated between April 2003 and May 2015, 35 patients underwent segmental or marginal mandibulectomy. The use of radical resection was based on clinical and/or radiographic evidence of size, cortical perforation and subsequent soft tissue involvement, and on the history of previous recurrence of the same lesion. Recurrence, justifications of the main major factor for resection, and functional and cosmetic results of the patients following mandibular reconstruction were systematically evaluated. There were $26 \mathrm{OKCs}$ in the mandibular molar-ramus region, eight in the mandibular anterior-premolar region and one in the mandibular molar-ramus and anterior-molar regions. Among the 35 patients, 20 had primary OKCs and 15 had recurrent OKCs. A total of 31 patients underwent segmental mandibulectomy, of which 28 were immediately reconstructed with a vascularized flap, whereas four patients underwent marginal mandibulectomy. The functional and cosmetic outcomes of patients were evaluated as satisfactory. The length of the follow-up period ranged from 2 to 17 years following operation (average, 5.82 years). Recurrence was identified in one patient who had been treated with marginal mandibulectomy. In conclusion, the findings from the present study suggested that radical resection may be recommended for patients with OKCs and locally aggressive features. Immediate mandibular reconstruction with a vascularized flap
\end{abstract}

Correspondence to: Professor Liu Bing, Department of Oral Maxillofacial Head and Neck Oncology Surgery, School and Hospital of Stomatology, Wuhan University, 237 Luoyu Road, Wuhan, Hubei 430079, P.R. China

E-mail: liubing9909@whu.edu.cn

Key words: radical resection, odontogenic keratocyst, mandibular reconstruction, recurrence, bone graft may be a crucial part of this aggressive treatment method that may reduce OKCs-associated morbidity.

\section{Introduction}

Keratocystic odontogenic tumor (KOT), which has recently been renamed as odontogenic keratocyst (OKC), is one of the most controversial pathologic entities in maxillofacial pathology (1-3). OKC is characterized by a thin and regular lining of parakeratinized stratified squamous epithelium with palisading hyperchromatic basal cells (1). The unique features of OKCs comprise a locally aggressive behavior with a high recurrence rate and a tendency to multiply $(4,5)$. Recurrence occurs following inadequate treatment methods, incomplete removal of the cyst or epithelial islands appearing after enucleation, a high mitotic index of the epithelial cells, large size of the cyst and a lesion location associated with difficult surgical access $(4,6,7)$.

The treatment method for OKCs remains controversial. To the best of our knowledge, there is no evidence in the literature that would guide surgeons in selecting the best treatment options. Clinicians continue to rely on their own experience when deciding for the most appropriate treatment. Surgical approaches vary between conservative to more aggressive treatments $(8,9)$. Enucleation is the most commonly used treatment method (6) and is associated with a high recurrence rate $(10,11)$. Decompression and marsupialization has been used as a conservative treatment of OKCs (12). Some clinicians do not approve these techniques because the potential remnant cystic tissues left behind can facilitate the recurrence (13). A more aggressive approach may therefore lower the risk of recurrence (14). It has been suggested that aggressive resection should be limited to OKCs that have recurred more than twice or to those that have undergone malignant transformation $(4,15)$. In addition, Worrall (16) recommended radical excision as the treatment of choice for OKCs that had cortically perforated, whereas Gosau et al (17) reported that enucleation plus curettage with carnoy's solution results in a recurrence rate statistically comparable to that of resection excision. Jackson et al (18) highlighted that complete excision should be performed for OKCs associated with any sign of soft tissue involvement. There is therefore high variability 
in the decision-making process for the resection of OKCs. Although radical resection offers the highest cure rate, it produces significant morbidity, including the loss of jaw continuity or facial disfigurement. Evaluation of patients following such aggressive treatment is therefore necessary. The present study reported the clinical outcomes of 35 patients who were treated with radical resection followed by immediate defect reconstruction.

\section{Materials and methods}

Patients. A retrospective review of well-documented cases of OKCs that were treated between April 2003 and May 2015 at the Department of Oral and Maxillofacial Surgery of the School and Hospital of Stomatology, Wuhan University was conducted. The involvement sites of the lesions were divided into the mandibular anterior-premolar and the mandibular molar-ramus regions. Data were obtained from the pathological department and comprised the orthopantomogram radiographs or $\mathrm{CT}$ scans performed prior to and following the treatment, and medical record notes. Patients were excluded if the data records were incomplete, if the follow-up period was $\leq 12$ months or if they had multiple cysts that were associated with nevoid basal cell nevus syndrome.

Treatment procedures. The treatment plans were based on clinical or radiographic evidence of cortical perforation of the inferior or posterior mandibular borders and subsequent soft tissue involvement, multilocular large cysts and a history of previous recurrence of the same lesion. All cases associated with any of these features were considered to have a more aggressive behavior and were therefore treated with radical resection, followed by immediate reconstruction of the defect with autogenous bone grafts (Fig. 1). In contrast, marginal mandibulectomy was used with $\sim 1 \mathrm{~cm}$ margin around the lesion, whereas the lower border of the mandible and/or the posterior border of the ramus was maintained intact (Fig. 2). When the soft tissue adjacent to the cyst was involved, excision of the soft tissue up to the next anatomical boundary was recommended and the excised tissue was frozen and observed to determine adequacy of surgical margins. Resection and reconstruction procedures were performed via transmandibular approach with general anesthesia and naso-endotracheal intubation. Two surgical teams simultaneously operated on these patients, for whom immediate defect reconstruction was performed. One team performed the mandible resection and prepared the recipient site for flap transfer, whereas the other team handled the vascularized flap. The length of the follow-up period for patients that underwent radical resection followed by reconstruction ranged from 2 to 17 years following surgery (average, 5.82 years).

The justifications for resection were systematically and carefully analyzed to identify the common major factor. The functional results of patients with mandibular reconstruction were evaluated using a self-assessment questionnaire that was completed 6 months after surgery. The questionnaire consisted in questions regarding social activities, diet, speech, oral continence and facial appearance. The cosmetic appearance following reconstruction was assessed by the patients (patients perception) using their own pre and post-reconstruction photographs (frontal and/or profile views) and also by two experienced doctors. Postoperative complications, including plate breakage, plate exposure, failure of the flap, infection and recurrence were also evaluated. The judgement criteria were the presence or absence of recurrence, jaw function and cosmetic outcome.

Statistical analysis. Descriptive statistics, including frequency distribution and percentage, measures of central tendency (average, median) and measures of dispersion (range) were used to report the data. The questionnaire was approved by the Medical Ethic Committee of the School and Hospital of Stomatology, Wuhan University, and written informed consent was obtained from all patients.

\section{Results}

Clinical characteristics of patients. The present retrospective study evaluated 565 patients with OKCs. A total of 43 patients treated with a radical approach were selected. Among them, two patients diagnosed with basal cell nevus syndrome, one patient with a primary OKC and $\leq 12$ months follow-up, and five patients with OKCs that occurred in the maxilla were excluded. The remaining 35 patients (6.19\%) underwent OKCs resection. Table I presents the patient demographics, lesion characteristics, treatments and outcomes. The age of the patients ranged from 20 to 77 years old, with a median age of 39.6 years. There were 27 men $(77.14 \%)$ and eight women (22.86\%). Among the 35 patients, 26 had OKCs in the mandibular molar-ramus region (74.29\%), eight had OKCs in the mandibular anterior-premolar area (22.86\%) and one had OKCs in the mandibular molar-ramus and anterior-molar regions $(2.9 \%)$. A total of 18 patients experienced cortical perforation with subsequent soft tissue involvement or extension into the adjacent tissues. Furthermore, 20 patients had primary OKCs $(57.14 \%)$ and 15 patients experienced recurrent OKCs $(42.86 \%)$. Patients suffered from recurrent OKCs for 1-34 years prior to resection (average, 8.8 years). The size of the OKCs varied from 7 to $15 \mathrm{~cm}$, with an average size of $9.40 \mathrm{~cm}$.

Treatment. Segmental mandibulectomy was completed in 31 patients $(88.57 \%)$. Following resection, 28 patients $(90.32 \%)$ were immediately reconstructed with a vascularized fibula flap (11 patients), a vascularized iliac crest flap (16 patients) or frozen autogenous bone replantation (one patient). No reconstruction was completed in three patients due to economic constraints. Marginal mandibulectomy was performed in four patients $(11.43 \%)$, and one patient underwent immediate reconstruction with an alloplastic plate to prevent postoperative pathological fracture. In total, 18 patients had $7-9 \mathrm{~cm}$ in length of bone harvested, whereas 10 patients had $10-15 \mathrm{~cm}$ in length of bone harvested.

Functional outcomes. The functional outcomes of patients were evaluated and the results revealed that diet and oral continence were normal, speech was easily understood for all patients, but social activities were diminished for three patients. Following mandibular reconstruction, no patients experienced complications of flap failure, plate exposure or 

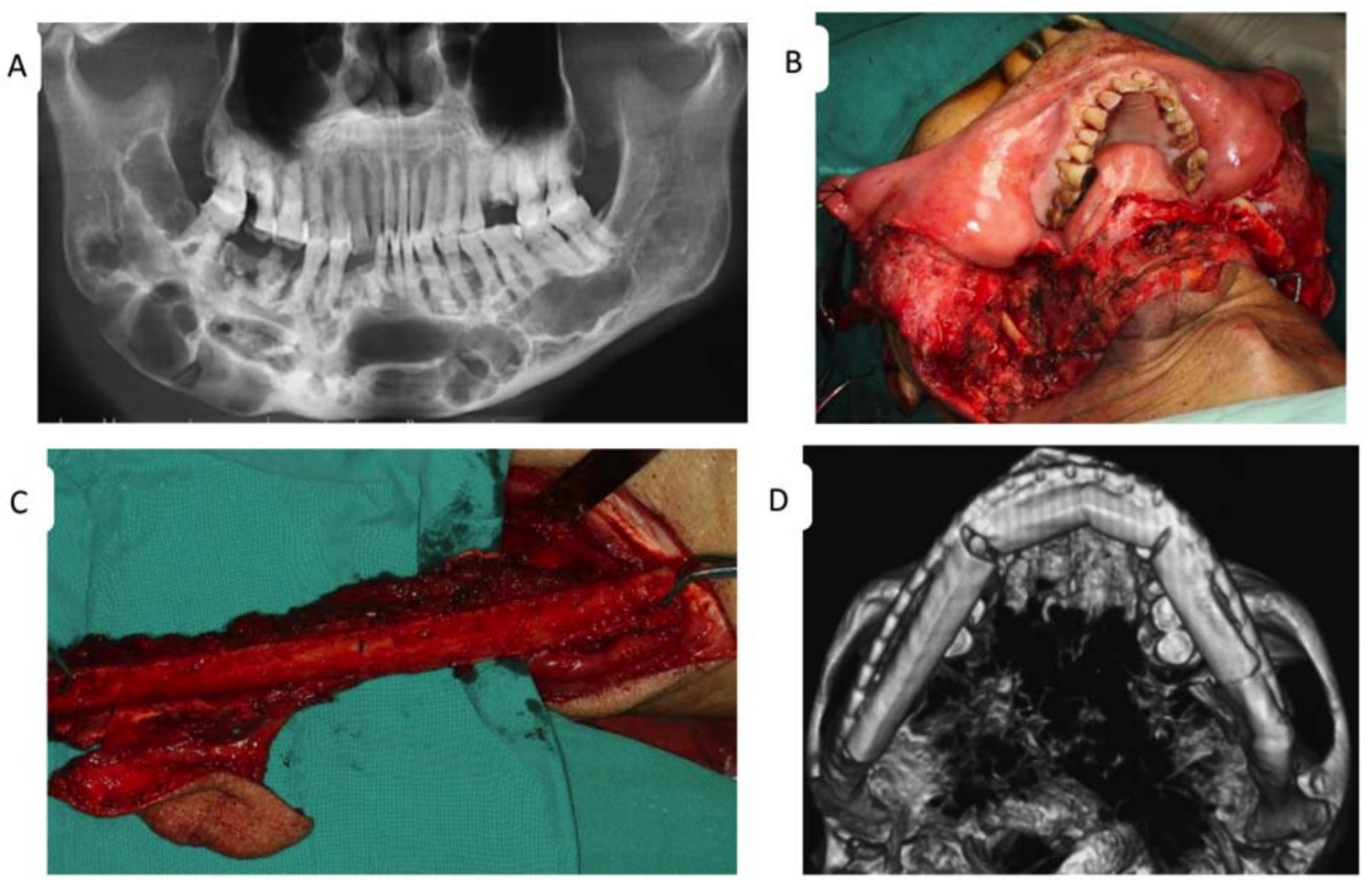

Figure 1. Features of odontogenic keratocyst determined the treatment regimen. (A) A preoperative panoramic radiograph of a large lesion with multiple radiolucencies extending from the left third molar to the contralateral ascending branch of the mandible. Intraoperative views of (B) a segmental mandibulectomy and (C) the fibula flap. (D) An image of the reconstructed mandible following surgery.
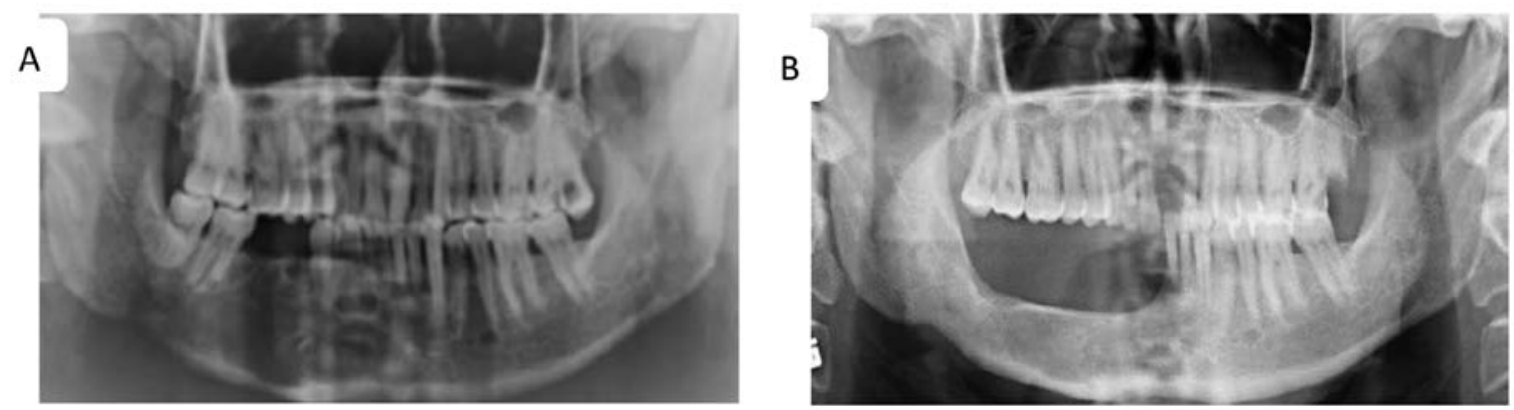

Figure 2. Marginal mandibulectomy of recurrent keratocysts of the right mandible. (A) Panoramic radiograph of multiple radiolucencies with a thin sclerotic line on the alveolar bone of the right mandibular anterior teeth, the second premolar and the first molar extraction sites, indicating recurrences. (B) Panoramic radiograph captured 18 months following marginal mandibulectomy.

plate breakage. Three patients experienced moderate infections that were treated with local wound care and antibiotic (Table II). The facial appearance of all patients following surgical intervention was evaluated as good by the surgeons, and six of the patients rated their facial appearance as acceptable (Fig. 3). There was one recurrence in a patient treated with marginal mandibulectomy after a period of 5 years follow-up.

\section{Discussion}

The understanding of OKCs treatment modalities has increased over the past few decades; however, the outcome remains controversial. Enucleation is the most commonly used method for surgical treatment of OKCs, although it presents a significantly higher recurrence rate compared with other treatment options $(4,6,19)$. Since OKCs lining is typically thin and friable, cyst removal in one piece can therefore be difficult, in particular for large OKCs with multilocular lesions. The use of adjunctive therapies usually follows enucleation and reduces the recurrence rate to $\leq 10 \%$ (20). Decompression and marsupialization have been also used as a conservative treatment method and both are beneficial in certain cases (13). These two techniques offer the advantages of considerably reducing the cyst volume and diminishing the risks of injuries to certain structures, including teeth or inferior dental nerve (12). The OKC wall after marsupialization commonly undergoes histological and immunohistochemical changes and may transform into normal oral mucosa without inherent aggressive features (21), which could explain the lower recurrence rates in patients with OKCs treated by this approach (22). However, both methods require a longer treatment period with multiple-staged procedures. The high degree of OKCs 


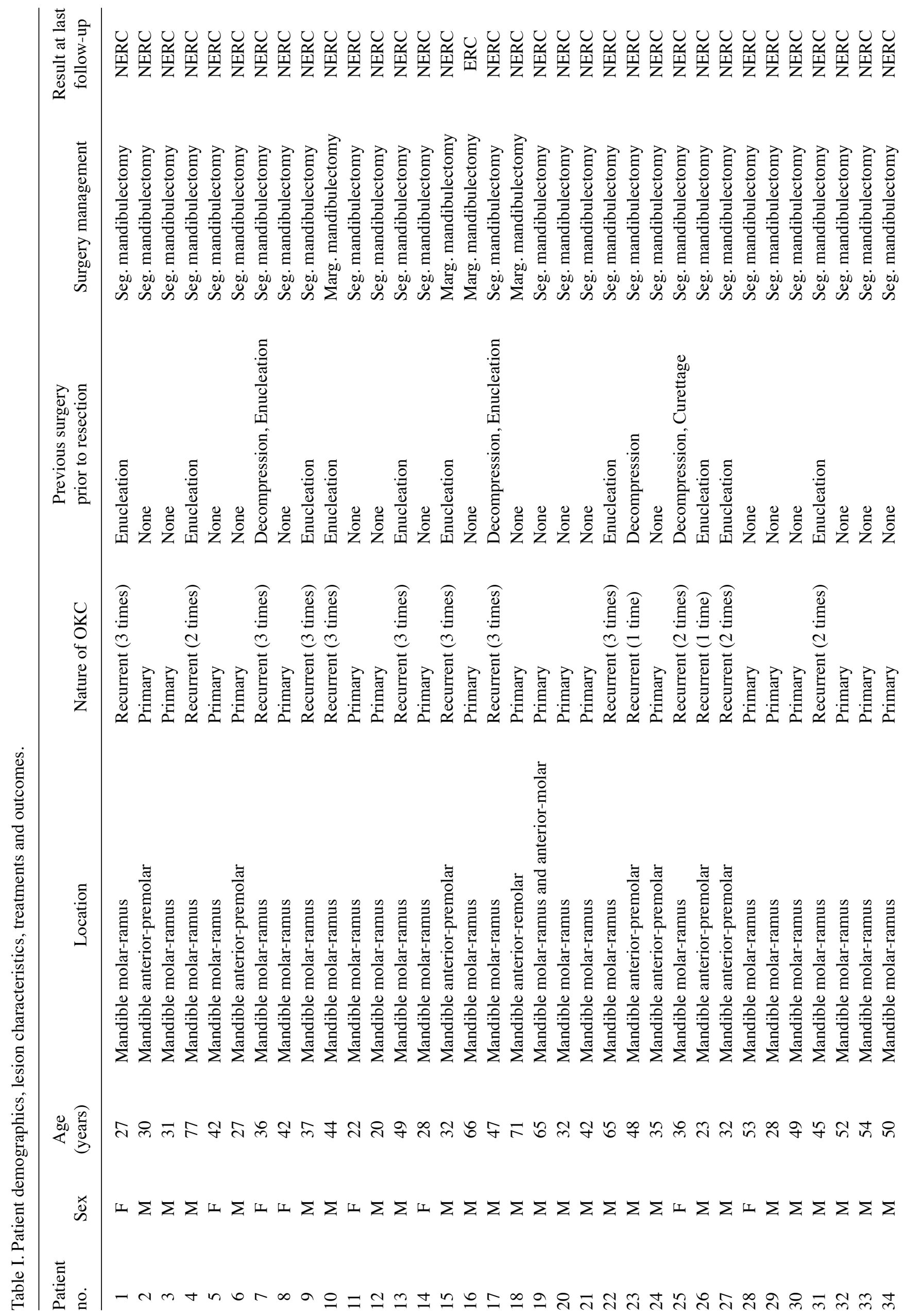


Table II. Complication, functional and esthetic outcomes of patients following treatment.

\begin{tabular}{|c|c|c|}
\hline Variables & $\begin{array}{c}\text { Mandibular } \\
\text { reconstruction }(\mathrm{n}=28)\end{array}$ & Percentage \\
\hline \multicolumn{3}{|l|}{ Diet } \\
\hline Normal & 28 & 100 \\
\hline Soft & - & - \\
\hline \multicolumn{3}{|l|}{ Speech } \\
\hline Easily understood & 28 & 100 \\
\hline Understood with effort & - & - \\
\hline Unintelligible & - & - \\
\hline \multicolumn{3}{|l|}{ Social activities } \\
\hline Normal & 25 & 89.29 \\
\hline Diminished & 3 & 10.71 \\
\hline \multicolumn{3}{|l|}{ Oral continence } \\
\hline Norma & 28 & 100 \\
\hline Slight/severe drooling & - & - \\
\hline \multicolumn{3}{|l|}{ Facial appearance } \\
\hline \multicolumn{3}{|l|}{ Excellent/good } \\
\hline Patient & 24 & 85.71 \\
\hline Surgeon team & 28 & 100 \\
\hline \multicolumn{3}{|l|}{ Acceptable } \\
\hline Patient & 6 & 21.43 \\
\hline Surgeon team & - & - \\
\hline \multicolumn{3}{|l|}{ Poor } \\
\hline Patient & - & - \\
\hline Surgeon team & - & - \\
\hline \multicolumn{3}{|l|}{ Complications } \\
\hline Flap failure & 0 & 0 \\
\hline Plate exposure & 0 & 0 \\
\hline Plate breakage & 0 & 0 \\
\hline Infection & 3 & 7.7 \\
\hline
\end{tabular}

recurrence explains the inclination of certain surgeons to eliminate the disease through a single radical surgical approach; however the morbidity associated with this radical approach guides other clinicians to select more conservative techniques. Well-conducted randomized controlled clinical trials analyzing the management of OKCs are therefore required. To the best of our knowledge, no conclusions on the effectiveness of the treatments for OKCs have yet been made (23) and there is no strong evidence that can guide surgeons to select the best treatment option. In the present study of 565 patients with OKCs, only 35 patients (6.19\%) underwent segmental or marginal resection, and immediate reconstruction was performed in 28 of them (93.32\%). Based on the aforementioned results, a protocol for managing OKCs was established (Fig. 4). Segmental resection should be considered in the following situations: i) Primary OKC with any radiologic appearance, exhibiting aggressive clinical behavior such as multiple perforations of the jaws cortical plates or extension into adjacent tissues, the involvement of any location (particularly in the mandibular molar-ramus region), and multilocular 
A

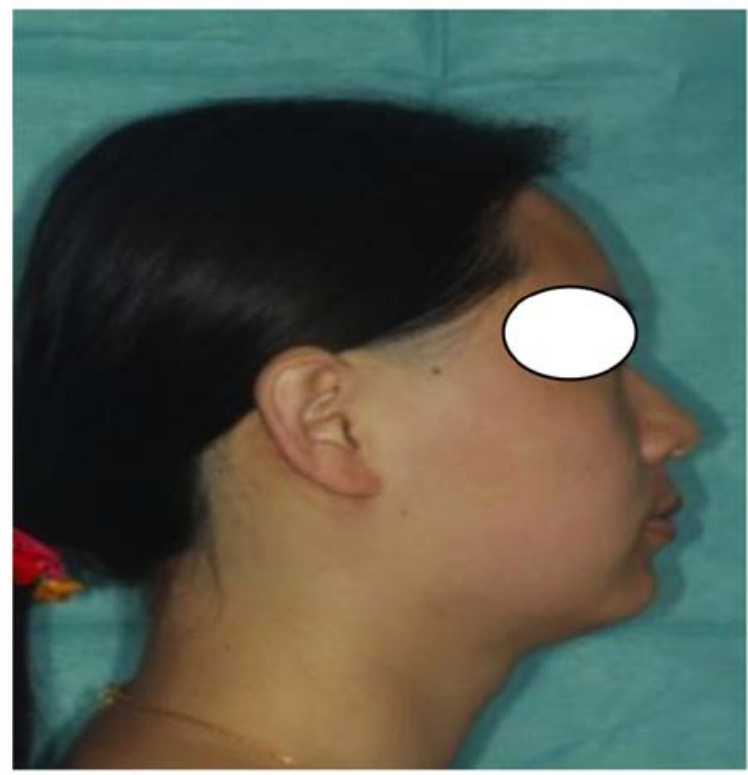

B

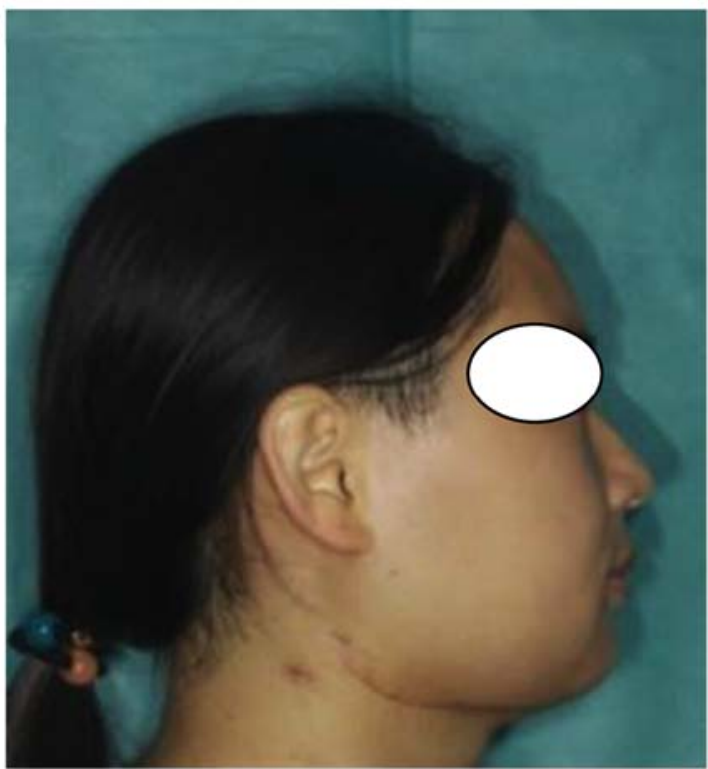

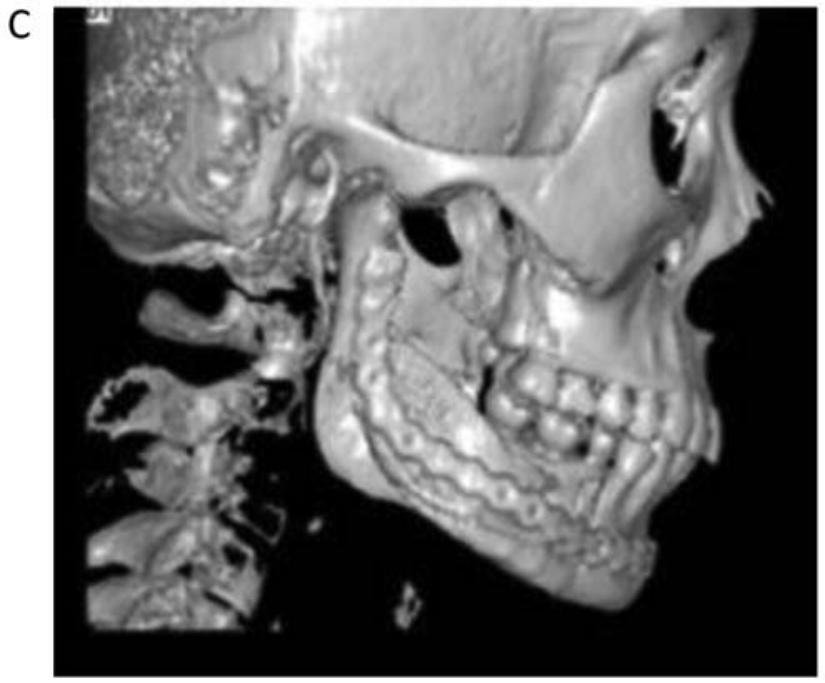

Figure 3. Postoperative outcome evaluation of facial appearance. (A) Profile photographs before and (B) after surgery. (C) An image of the reconstructed mandible at 6 months following operation.

large lesions; and ii) recurrent OKC with or without cortical perforation, or failure to comply with the interval time follow-up schedule and with a size either similar or larger than the primary lesion, particularly the multiple recurrent $\mathrm{OKCs}$ in any location. If the primary lesion or recurrent lesion with multiple radiolucencies does not grow sizable or without the thinned inferior border of the mandible) and is located in the mandible anterior region, marginal mandibulectomy is preferred.

Previous studies have demonstrated that mandibular molar-ramus is the most common region with frequent perforation of the overlying bone by OKCs and with firm cysts adhesion to the overlying mucosa $(19,20,24,25)$. Excision of the overlying mucosa with the cysts and curettage with carnoy's solution or electrocautery has been recommended in this area (20). However, conservative treatment methods are inadequate when OKCs have extended and involve muscles with cortical perforation $(18,26)$. In addition, OKCs that occur in the mandibular molar-ramus region have significantly higher recurrence rates $(75 \%)$ compared with those in other sites (27). Perforation of the cortical plates and extension of the lesions from the masseter, as presented in patient no. 35 of the present study, or extension of the lesions from the pterygoid or the temporalis muscle to the infratemporal fossa or orbit-skull base are possible at this stage, and elimination of these OKCs requires a heavy wide surgery. The thinned inferior border does not serve any purpose as chances of pathological fracture occur. Subsequently, it is may be crucial to treat primary OKC associated with aggressive behaviors more radically in order to avoid a severe recurrent lesion.

Most studies emphasize that radical resection should be limited to multiple recurrent $\mathrm{OKCs}$, or to those that have undergone malignant transformation $(4,15,28)$. Worrall $(16)$ and Jackson et al (18) recommended radical excision as the treatment of choice for OKCs with bone perforation or unpredictable lesions with any signs of soft tissue involvement. Ahlfors et al (29) suggested that in order to reduce the high recurrence rate of OKCs, surgery should include marginal resection, including a rim of uninvolved bone, which is similar to the treatment suggested in cases of unicystic ameloblastoma. 


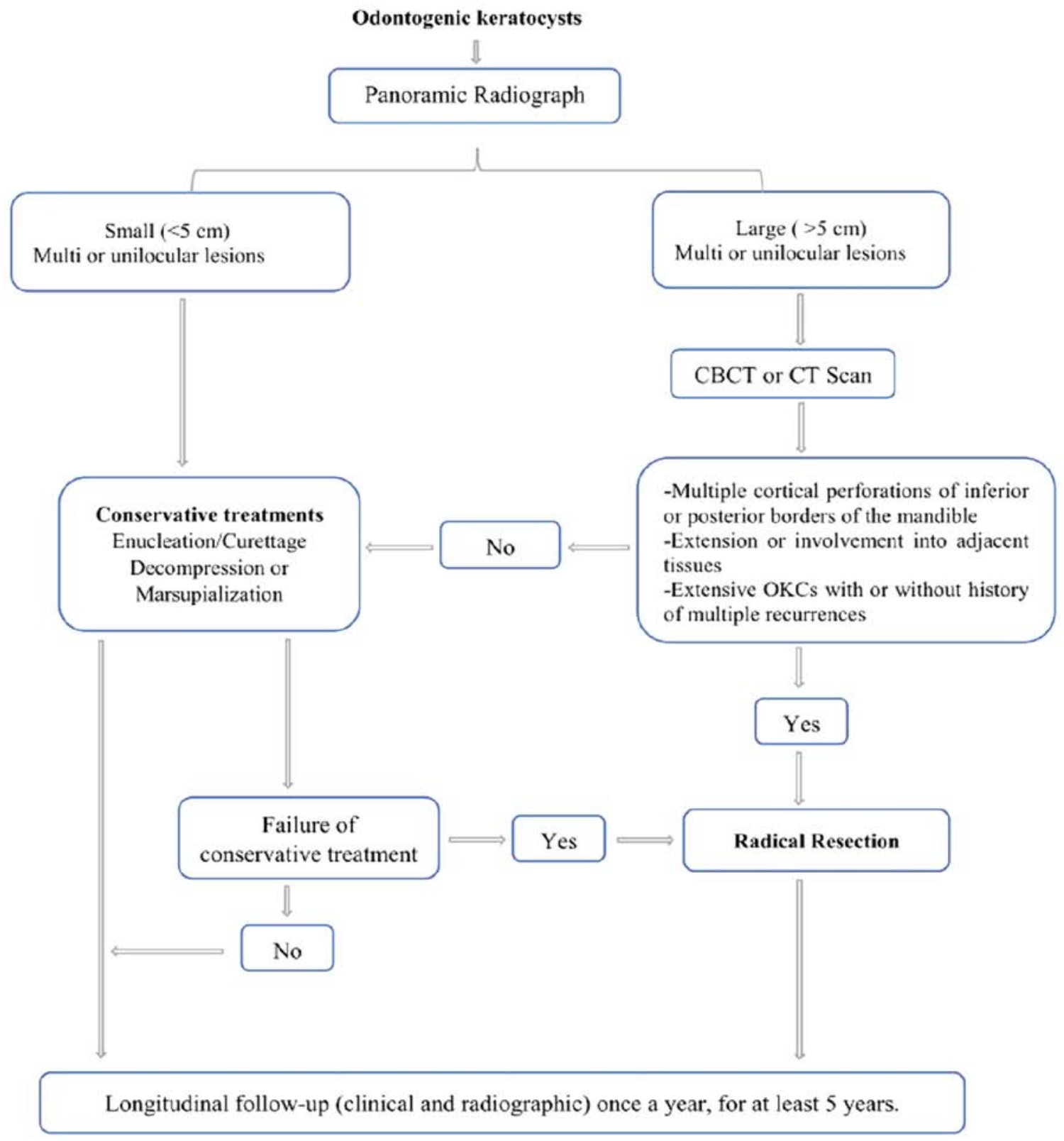

Figure 4. Clinical protocol managing OKCs of the jaws. CBCT, cone beam CT; CT, computed tomography; OKCs, odontogenic keratocysts.

In the present study, the pathological sign that influenced the decision to perform radical resection was primary and recurrent $\mathrm{OKCs}$ that had clinically aggressive behavior and that mostly occurred in the mandible molar-ramus region. Segmental or marginal mandibulectomy was performed for those OKCs, which was in agreement with previous studies that demonstrated that $\sim 100 \%$ of recurrent OKCs contain epithelial islands and microcysts in the overlying mucosa areas $(20,30)$. The absence of microcysts has been demonstrated in the surrounding bone following block resections of recurrent OKCs (30).

OKCs recurrence within the bone graft following segmental resection has also been reported $(6,24,26)$. The involvement of the surrounding soft tissues is common in mandibular molar-ramus $\mathrm{OKCs}$, and the bone perforation caused by $\mathrm{OKCs}$ ranges from 39 to $50.8 \%$ (6), which was also demonstrated in the present study. This may be considered as the main cause of recurrence in patients following marginal mandibulectomy observed in the present study. The presence of multifocal cysts disease may also explain the recurrence rate. Stoelinga (20) reported 3/80 patients and Boyne et al (31) reported seven cases with multiple recurrent OKCs, of which six underwent segmental mandibulectomy over 10-21 years. Their histologic examination revealed cyst formation at distant sites from the original cyst in all cases, which suggest that the lesions were multifocal. There are numerous confounding factors associated with the recurrence and clinicopathologic behavior of OKCs (13). Surgical management is the most influential factor on recurrence. In the present study, one patient with $\mathrm{OKC}$ that involved deep surface of the masseter muscle after segmental mandibulectomy had also been reported in our previous study (24).

The major sequelae of radical resection affect the cosmetic and functional outcomes. Recently, the development of free vascularized bone graft has facilitated the decision-making process for surgical ablation in oral and maxillofacial surgery, 
and has led to a high success rate of $\sim 100 \%$ (32). In the present study, a large number of patients who underwent segmental mandibulectomy were immediately reconstructed with a vascularized flap, which was not the case in our previous study (19). Immediate reconstruction with a vascularized flap allowed good healing and reduced the risk of bone resorption. Free fibula and iliac crest flaps were the most widely used techniques in the present study, as also reported in a previous study (33). The fibula flap is the only donor site that allows reconstruction of entire mandibular defects. The free iliac crest flap offers more choices for mandible reconstruction when the length of bone harvested is $\leq 8 \mathrm{~cm}$, in particular when used for reconstruction of the mandibular body. In the present study, most patients were greatly satisfied by their treatment, with regards to resumption of normal diet, good speech, oral continence and good facial appearance.

Conservative treatment methods of $\mathrm{OKC}$ are still preferred by most surgeons. There is ongoing debate over the preservation structure and reduction of the recurrence, and numerous surgical approaches have been applied to OKCs. The understanding of OKC treatment is clear to some extent; the overall recurrence rate of $\leq 20 \%$ is not discouraging and there is a low recurrence rate following resection.

The present study demonstrated that radical resection was only suitable for OKCs with locally aggressive features. Immediate reconstruction of the defect with vascularized fibula or crest iliac flaps allowed positive functional and cosmetic outcomes for patients who underwent an aggressive treatment. Multiples recurrent lesions and primary OKCs with aggressive clinical behavior, including perforation of the cortical plates of the jaws or extension into the adjacent tissues, extensive lesions, and presence in the mandibular molar-ramus region were the main factors in the decision-making process for radical resection. When possible, it was acceptable to start with a conservative approach, including marsupialization followed by enucleation, in particular in young patients or elderly patients who are medically compromised. Long-term follow-up remains important to monitor possible disease recurrence.

\section{Acknowledgements}

Not applicable.

\section{Funding}

No funding was received.

\section{Availability of data and materials}

The datasets used and/or analyzed during the current study are available from the corresponding author on reasonable request.

\section{Authors' contributions}

NBF contributed to the design of the study and wrote the manuscript. NBF, YS, TW and YYZ collected the clinical samples. YFZ, TW and LB performed the data analysis and revised the manuscript. All authors have read and approved the manuscript.

\section{Ethics approval and consent to participate}

The questionnaire survey was approved by The Medical Ethic Committee of the School and Hospital of Stomatology, Wuhan University (Hubei, China). Written informed consent was obtained from the patients.

\section{Patient consent for publication}

Written informed consent was obtained from the patients.

\section{Competing interests}

The authors declare that they have no competing interests.

\section{References}

1. Speight PM and Takata T: New tumour entities in the 4th edition of the World Health Organization Classification of Head and Neck tumours: Odontogenic and maxillofacial bone tumours. Virchows Arch 472: 331-339, 2018.

2. Philipsen HP: Pathology and Genetics of Head and Neck Tumours. World Health Organization Classification of Tumours. Barnes L, Eveson JW, Reichart P and Sidransky D (eds). IARC, Lyon, pp306-307, 2005

3. Peacock ZS: Controversies in oral and maxillofacial pathology. Oral Maxillofac Surg Clin North Am 29: 475-486, 2017.

4. Al-moraissi EA, Dahan AA, Alwadeai MS, Oginni FO, Al-jamali JM, Alkhutari AS, Al-Tairi NH, Almaweri AA and Al-Sanabani JS: What surgical treatment has the lowest recurrence rate following the management of keratocystic odontogenic tumor?: A large systematic review and meta-analysis. J Craniomaxillofac Surg 45: 131-144, 2017.

5. Pitak-Arnnop P, Chaine A, Oprean N, Dhanuthai K, Bertrand JC and Bertolus C: Management of odontogenic keratocysts of the jaws: A ten-year experience with 120 consecutive lesions. J Craniomaxillofac Surg 38: 358-364, 2010.

6. Chirapathomsakul D, Sastravaha P and Jansisyanont P: A review of odontogenic keratocysts and the behavior of recurrences. Oral Surg Oral Med Oral Pathol Oral Radiol Endod 101: 5-9, 2006.

7. Yagyuu T, Kirita T, Sasahira T, Moriwaka Y, Yamamoto K and Kuniyasu H: Recurrence of keratocystic odontogenic tumor: Clinicopathological features and immunohistochemical study of the Hedgehog signaling pathway. Pathobiology 75: 171-176, 2008.

8. Sánchez-Burgos R, González-Martín-Moro J, Pérez-Fernández E and Burgueño-García M: Clinical, radiological and therapeutic features of keratocystic odontogenic tumours: A study over a decade. J Clin Exp Dent 6: e259-e264, 2014.

9. Al-Moraissi EA, Pogrel MA and Ellis E III: Enucleation with or without adjuvant therapy versus marsupialization with or without secondary enucleation in the treatment of keratocystic odontogenic tumors: A systematic review and meta-analysis. J Craniomaxillofaciac Surg 44: 1395-1403, 2016.

10. Zecha JA, Mendes RA, Lindeboom VB and van der Waal I: Recurrence rate of keratocystic odontogenic tumor after conservative surgical treatment without adjunctive therapies-A 35 -year single institution experience. Oral Oncol 46: 740-742, 2010.

11. Cunha JF, Gomes CC, de Mesquita RA, Andrade Goulart EM, de Castro WH and Gomez RS: Clinicopathologic features associated with recurrence of the odontogenic keratocyst: A cohort retrospective analysis. Oral Surg Oral Med Oral Pathol Oral Radiol 121: 629-635, 2016.

12. Pogrel MA and Jordan RC: Marsupialization as a definitive treatment for the odontogenic keratocyst. J Oral Maxillofac Surg 62: 651-655; discussion 655-656, 2004.

13. Mendes RA, Carvalho JF and van der Waal I: Characterization and management of the keratocystic odontogenic tumor in relation to its histopathological and biological features. Oral Oncol 46: 219-225, 2010.

14. Blanas N, Freund B, Schwartz M and Furst IM: Systematic review of the treatment and prognosis of the odontogenic keratocyst. Oral Surg Oral Med Oral Pathol Oral Radiol Endod 90: 553-558, 2000. 
15. Kolokythas A, Fernandes RP, Pazoki A and Ord RA: Odontogenic keratocyst: To decompress or not to decompress? A comparative study of decompression and enucleation versus resection/peripheral ostectomy. J Oral Maxillofac Surg 65: 640-644, 2007.

16. Worrall SF: Recurrent odontogenic keratocyst within the temporalis muscle. Br J Oral Maxillofac Surg 30: 59-62, 1992.

17. Gosau M, Draenert FG, Müller S, Frerich B, Bürgers R, Reichert TE and Driemel O: Two modifications in the treatment of keratocystic odontogenic tumors (KCOT) and the use of carnoy's solution (CS)-a retrospective study lasting between 2 and 10 years. Clin Oral Investig 14: 27-34, 2010.

18. Jackson IT, Potparic Z, Fasching M, Schievink WI, Tidstrom K and Hussain K: Penetration of the skull base by dissecting keratocyst. J Craniomaxillofac Surg 21: 319-325, 1993.

19. Zhao YF, Wei JX and Wang SP: Treatment of odontogenic keratocysts: A follow-up of 255 Chinese patients. Oral Surg Oral Med Oral Pathol Oral Radiol 94: 151-156, 2002.

20. Stoelinga PJ: Long-term follow-up on keratocysts treated according to a defined protocol. Int J Oral Maxillofac Surg 30 $14-25,2001$.

21. August M, Faquin WC, Troulis MJ and Kaban LB: Dedifferentiation of odontogenic keratocyst epithelium after cyst decompression. J Oral Maxillofac Surg 61: 678-683; discussion 683-684, 2003.

22. Chrcanovic BR and Gomez RS: Recurrence probability for keratocystic odontogenic tumors: An analysis of 6427 cases. J Craniomaxillofac Surg 45: 244-251, 2017.

23. Sharif FN, Oliver R, Sweet C and Sharif MO: Interventions for the treatment of keratocystic odontogenic tumours. Cochrane Database Syst Rev CD008464, 2015.

24. Liu B, Cai Y, Wang SP and Zhao YF: Recurrent keratocystic odontogenic tumor in the masseter muscle overlying the boney perforations: A case report. Oral Surg Oral Med Oral Pathol Oral Radiol 113: e1-e5, 2012.
25. Stoelinga PJ: Etiology and pathogenesis of keratocysts. Oral Maxillofac Surg Clin North Am 15: 317-324, 2003.

26. Warburton G, Shihabi A and Ord RA: Keratocystic odontogenic tumor (KCOT/OKC)-clinical guidelines for resection. J Maxillofac Oral Surg 14: 558-564, 2015.

27. Myoung H, Hong SP, Hong SD, Lee JI, Lim CY, Choung PH, Lee JH, Choi JY, Seo BM and Kim MJ: Odontogenic keratocyst: Review of 256 cases for recurrence and clinicopathologic parameters. Oral Surg Oral Med Oral Pathol Oral Radiol Endod 91: 328-333, 2001.

28. Giuliani M, Grossi GB, Lajolo C, Bisceglia M and Herb KE: Conservative management of a large odontogenic keratocyst: Report of a case and review of the literature. J Oral Maxillofac Surg 64: 308-316, 2006

29. Ahlfors E, Larsson A and Sjögren S: The odontogenic keratocyst: A benign cystic tumour? J Oral Maxillofac Surg 42: 10-19, 1984.

30. Stoelinga PJ and Bronkhorst FB: The incidence, multiple presentation and recurrence of aggressive cysts of the jaws. J Craniomaxillofac Surg 16: 184-195, 1988.

31. Boyne PJ, Hou D, Moretta C and Pritchard T: The multifocal nature of odontogenic keratocysts. J Calif Dent Assoc 33: 961-965, 2005

32. Hidalgo DA and Pusic AL: Free-flap mandibular reconstruction A 10-year follow-up study. Plast Reconstr Surg 110: 438-449, discussion 450-451, 2002.

33. Lyons AJ, James R and Collyer J: Free vascularised iliac crest graft: An audit of 26 consecutive cases. Br J Oral Maxillofac Surg 43: 210-214, 2005.

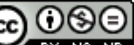

This work is licensed under a Creative Commons Attribution-NonCommercial-NoDerivatives 4.0 International (CC BY-NC-ND 4.0) License. 